\title{
DISCRETENESS PROPERTIES OF TRANSLATION NUMBERS IN SOLVABLE GROUPS
}

\author{
GREGORY R. CONNER
}

\begin{abstract}
We define a group to be translation proper if it carries a leftinvariant metric in which the translation numbers of the non-torsion elements are nonzero and translation discrete if they are bounded away from zero. The main results of this paper are that a translation proper solvable group of finite virtual cohomological dimension is metabelian-by-finite, and that a translation discrete solvable group of finite virtual cohomological dimension, $m$, is a finite extension of $\mathbb{Z}^{m}$.
\end{abstract}

\section{INTRODUCTION}

The notion of a translation number comes from Riemannian geometry. Suppose $M$ is a compact Riemannian manifold of nonpositive sectional curvature and $\widetilde{M}$ is its universal cover, enjoying the metric induced from $M$. Then the covering transformation group acts as isometries on $\widetilde{M}$. Under these conditions any covering transformation of $\widetilde{M}$ fixes a geodesic line. Since a geodesic line is isometric to $\mathbb{R}$, a covering transformation must translate each point of such a fixed geodesic line by a constant amount which is called the translation number of the covering transformation. This notion can be abstracted. Let $G$ be a group which is equipped with a metric, $d$, which is invariant under left multiplication by elements of $G$, i.e., $d(x y, x z)=d(y, z) \forall x, y, z \in G$. We then say that $d$ is a left-invariant group metric (or a metric for brevity) on $G$. Let \|\|$: G \longrightarrow \mathbb{Z}$ be defined by $\|x\|=d\left(x, 1_{G}\right)$. To fix ideas, one may consider a finitely generated group equipped with a word metric.

For $x$ in $G$, let $\tau(x)=\lim \sup _{n \rightarrow \infty} \frac{\left\|x^{n}\right\|}{n}$. This quantity is called the translation number of $x$. We define a group to be translation discrete if it carries a metric in which the translation numbers of the non-torsion elements are bounded away from zero. Similarly a group is called translation proper if it admits a metric so that only torsion elements have zero translation number.

There are several interesting applications of translation numbers to geometric and combinatorial group theory in the literature, Gersten and Short [GS] use translation numbers to prove results about subgroups of biautomatic groups, Gromoll and Wolf $[\mathrm{GW}]$ use translation numbers in the proof of their celebrated theorem. Gromov [Gr, BGS], Busemann [Bu] and others have used also translation numbers in their work. For instance, it is well-known that a group acting discretely cocompactly on a $\operatorname{CAT}(0)$ space is translation discrete, see [C1]. Putting this fact together with the main result we can get a mild generalization of the Gromoll/Wolf [GW] - Lawson/Yau theorem, namely that every solvable subgroup of a cocompact group of isometries of a CAT(0) space of finite cohomological dimension is abelianby-finite. Recently Bestvina [B] has used the results in the current article to study Artin groups. 
This goal of this paper is to discuss the following question : What kind algebraic information can one determine from the translation numbers of a solvable group? We will discuss the specific results of this paper. We prove a number of elementary results on left-invariant group metrics. We show that a translation proper solvable group of finite virtual cohomological dimension is metabelian-by-finite. An example is given of a polycyclic group which is translation proper, but not translation discrete. We prove that a translation discrete solvable group of finite virtual cohomological dimension, $m$, is a finite extension of $\mathbb{Z}^{m}$. To prove the necessity of the cohomological dimension hypothesis in the preceding result an example is given of a nonabelian solvable group which is translation discrete.

\section{Preliminaries}

Definition 2.1 (Metric, Norm). If $G$ is a group, we say that a non-negative realvalued function, $d$, on $G \times G$ is a semimetric on $G$ if it is symmetric, left-invariant under the action of $G$ on itself, satisfies the triangle inequality and $d\left(1_{G}, 1_{G}\right)=0$. We call $d$ a metric on $G$ if in addition it is definite (i.e. $d(g, h)=0 \Rightarrow g=h$ ). A (semi)metric on a group $G$ induces a (semi)norm on $G$ by defining $\|g\|=d\left(g, 1_{G}\right)$ for all $g \in G$. Similarly, if one is given a (semi)norm on a group $G$ one can define a (semi)metric on $G$ by defining $d(g, h)=\left\|h^{-1} g\right\|$.

If $\mathrm{G}$ is a group with a given (semi)metric and $\mathrm{H}$ is a subgroup of $\mathrm{G}$, then restricting the (semi)metric of $\mathrm{G}$ to $\mathrm{H}$ gives a (semi)metric of $\mathrm{H}$. We call a metric, $d$, on a group $G$ discrete if it induces the discrete topology on $G$.

Given a finitely generated group $G$ and a finite generating set $\gamma$ one can define a norm on $G$ by assigning to every element of $\mathrm{G}$ the length of the shortest word in the generators and their inverses which represents it. We shall call this the word norm on $G$ corresponding to $\gamma$. The associated metric is called the word metric on $G$ corresponding to $\gamma$. A word norm on $G$ has the property that it is, by construction, maximal among all nonnegative real-valued functions on $G$ satisfying the triangle inequality and taking values of at most 1 on the generating set.

Definition 2.2 (Translation number). Let $x$ be an element of a group $\mathrm{G}$ with seminorm \|\| . We define the translation number of $x$ as follows:

$$
\tau_{G}(x)=\liminf _{n \rightarrow \infty} \frac{\left\|x^{n}\right\|}{n} .
$$

Clearly, any element of finite order has translation number zero in any seminorm.

Definition 2.3 (Translation Discrete, Translation Proper). A semimetric (or the corresponding seminorm) on a group is said to be to be translation discrete if the translation numbers of the nontorsion elements of the group with respect to that semimetric are bounded away from zero. We will say that a group is translation discrete if it admits a semimetric which is translation discrete. A semimetric (seminorm) is said to be translation proper if the translation numbers of the nontorsion elements of the group with respect to that semimetric are nonzero. A group is translation proper if it admits a semimetric which is translation proper.

We will use the following lemma implicitly throughout this article.

Lemma 2.4 ([C2], Lemma 2.6.1). If $G$ is a finitely generated group, the following are equivalent:

1. $G$ is translation discrete(proper). 
2. G has a translation discrete(proper) subgroup of finite index.

3. Every subgroup of $G$ is translation discrete(proper).

4. There exists a word metric on $G$ that is translation discrete(proper).

5. Every generalized word metric on $G$ is translation discrete(proper).

\section{Main Results}

This section consists of a list of the principal results this the paper. The main body of the proofs are contained in later sections.

Theorem 3.1. A translation proper solvable group which has finite virtual cohomological dimension is metabelian-by-finite.

In Example 7.1 we examine a polycyclic group which is translation proper, but not translation discrete. This is the same group which we show in [C3] to have irrational translation numbers in every word metric.

Corollary 3.2. A solvable subgroup of finite virtual cohomological dimension of a biautomatic group is metabelian-by-finite.

Proof. It was proven (using slightly different notation) by Gersten and Short in [GS] that torsion-free biautomatic groups are translation proper.

Theorem 3.3. A translation proper solvable linear group is metabelian-by-finite.

The following result is similar to a result which is claimed by Gromov ([Gr]). Bruce Kleiner $([\mathrm{K}])$ has informed me that he has a proof of this result which uses the methods described in [CS].

Theorem 3.4. Every solvable subgroup of finite virtual cohomological dimension, $m$, in a translation discrete group is a finite extension of $\mathbb{Z}^{m}$.

In Example 7.2 we investigate a finitely generated torsion-free solvable group of infinite cohomological dimension that is translation discrete but is not abelian-byfinite.

Question 3.5. The group in Example 7.2 is not finitely presentable. Is there an example of a finitely presented solvable group which is translation-discrete, but not abelian-by-finite?

\section{Some Technical Background}

Definition 4.1. Suppose $H$ and $K$ are subgroups of $G$ which together generate $G$, and each is equipped with a semimetric. Then we can form the product semimetric on $G$ in the following way : for any string of the form

$$
w=h_{1} k_{1} h_{2} k_{2} \cdots h_{n} k_{n}, h_{i} \in H, k_{i} \in K
$$

where $h_{i} \neq 1, i>1$ and $k_{j} \neq 1, j<n$, we define the length of $w$ as

$$
l(w)_{G}=\sum_{i=1}^{n}\left\|h_{i}\right\|_{H}+\left\|k_{i}\right\|_{K}
$$

and we define the (semi)norm on $G$ as

$$
\|g\|_{G}=\inf \left\{l(w)_{G} \mid w \text { represents } g\right\} .
$$

By induction one can define the product semimetric on the subgroup generated by any finite number of semimetrized subgroups. 
Definition 4.2 (Generalized word (semi)norm). Suppose $\gamma=\left\{g_{1}, \cdots, g_{n}\right\}$ is a finite generating set for the group $G$ and $f: \gamma \rightarrow[0, \infty)$. We can define a seminorm on $\left\langle g_{i}\right\rangle$ by $\left\|g_{i}^{n}\right\|_{\left\langle g_{i}\right\rangle}=|n| \cdot f\left(g_{i}\right)$. We can now define a seminorm on $G$ by taking the product seminorm induced by the subgroups $\left\langle g_{i}\right\rangle$. One can think of this as giving each generator a weight and then defining the seminorm of a group element to be the minimum weighted length of words representing it. If $f$ is a positive function then the construction gives a norm. The generalized (semi)metric associated to this data is the (semi)metric associated to the above (semi)norm. We call the metric a generalized word metric if $f$ is a positive function. A word metric is a special case of a generalized word metric where $f$ is taken to be the constant 1 function.

Definition 4.3 (Partial order on real-valued functions). If $f$ and $g$ are two nonnegative real-valued functions with equal domains, we say that $g$ is smaller than $f$ (and write $g \preceq f$ ) if $\exists \lambda, \epsilon$ nonnegative reals, $\lambda \neq 0$ such that $g<\lambda f+\epsilon$. Suppose $\lambda, \epsilon$ are nonnegative reals, $\lambda \neq 0$. We say two nonnegative real-valued functions $f$ and $g$ with equal domain are $(\lambda, \epsilon)$-equivalent if $(1 / \lambda) f-\epsilon \leq g \leq \lambda f+\epsilon$. If we wish to omit the constants we may write $f \sim g$. Clearly $f \sim g \Leftrightarrow f \preceq g$ and $g \preceq f$.

Clearly the properties of being translation discrete or translation proper are preserved by semimetric equivalence.

Observation 4.4. A word norm on $G$ has the property that it is, by construction, maximal among all nonnegative real-valued functions on $G$ satisfying the triangle inequality and taking values of at most 1 on the generating set. Similarly a generalized word metric on $G$ has the property that it is maximal among all semimetrics on $G$ bounded by the prescribed values on the generating set. Thus, any two generalized word metrics on the same group are equivalent. If $H$ is a subgroup of finite index in the group $G$, then any generalized word norm on $H$ is equivalent to any generalized word norm on $G$ restricted to $H$.

We will need the following result of Gersten and Short [GS].

Theorem 4.5. Let $G$ be a group with seminorm \|\| . Let $x, y \in G$. Then the following are true:

1. $0 \leq \tau(x)=\lim _{n \rightarrow \infty} \frac{\left\|x^{n}\right\|}{n} \leq\|x\|$.

2. $\tau(x)=\tau\left(y^{-1} x y\right)$.

3. $\tau\left(x^{n}\right)=n \tau(x) \forall n \in \mathbb{N}$.

4. $\tau(x) \leq \frac{\left\|x^{n}\right\|}{n} \forall n \in \mathbb{N}$.

5. $\tau(x)=\tau\left(x^{-1}\right)$.

Corollary 4.6. Let $G$ be a group with seminorm \|\| . Let $x, y \in G$. The following facts are immediate:

1. If $x$ and $y$ commute then $|\tau(x)-\tau(y)| \leq \tau(x y) \leq \tau(x)+\tau(y)$.

2. If $\widehat{\|\|}$ is seminorm on $G$ such that \|\|$\preceq\|\|$ then $\tau_{G} \preceq \widehat{\tau}_{G}$.

3. If $\widehat{\|\|}$ is seminorm on $G$ which is $(\lambda, \epsilon)$-equivalent to \|\| , then $\tau_{G}$ and $\widehat{\tau}_{G}$ are $(\lambda, 0)$-equivalent.

Definition 4.7 (Relative (semi)norms). Let $\gamma: G \longrightarrow K$ be a homomorphism. Let \|\| be a (semi)norm on G. Then we can define a (semi)norm \|\|$_{\gamma}$ on $\operatorname{im}(\gamma)$ by $\|k\|_{\gamma}=\inf _{g \in \gamma^{-1}(k)}\{\|g\|\}$. Similarly, for any $H \unlhd G$ we can define a (semi)norm on $G / H$ by defining $\|g H\|_{G / H}=\inf _{x \in g H}\|x\|$. 
Evidently these constructions are equivalent, since in one direction we can take $\gamma: G \longrightarrow G / H$ to be the natural map, and in the other direction we can take $H=\operatorname{ker}(\gamma)$.

Observation 4.8. If $\gamma: G \longrightarrow K$ is a homomorphism then $\|g\| \geq\|\gamma(g)\|_{\gamma} \forall g \in G$. Similarly, if $H \unlhd G$ then $\tau_{G}(g) \geq \tau_{G / H}(g) \forall g \in G$.

\section{Semidirect Products}

If we have a group $G$ equipped with a semimetric and an automorphism $\phi$ of $G$, we may consider the product semimetric on $G \rtimes_{\phi} \mathbb{Z}$ since $\mathbb{Z}$ has a canonical word metric. In our case, $G$ will have the form $\mathbb{Z}^{n}$. If we choose a free basis for $\mathbb{Z}^{n}$ we may consider any automorphism of $\mathbb{Z}^{n}$ as a matrix in $\operatorname{GL}(n, \mathbb{Z})$. We may also think of it as a matrix in $\operatorname{GL}(n, F)$ where $F$ is $\mathbb{Q}, \mathbb{R}$ or $\mathbb{C}$. This allows us to consider embeddings of $\mathbb{Z}^{n} \rtimes_{\phi} \mathbb{Z}$ into $F^{n} \rtimes_{\phi} \mathbb{Z}$. In these spaces we will use the standard linear algebra techniques (eigenvalues, Jordan normal form, etc.) to understand the metric on our original space.

Observation 5.1. If the group $G$ is equipped with two equivalent semimetrics and $\phi \in \operatorname{Aut}(G)$, then the two induced product semimetrics on $G \rtimes_{\phi} \mathbb{Z}$ are equivalent, by Corollary A.4.

Definition 5.2 (Euclidean norm). Let $F$ be either $\mathbb{Z}$ or a subfield of $\mathbb{C}$. Let $\left\{v_{i}\right\}$ be a basis for $F^{n}$. Then each $x \in F^{n}$ can be written uniquely as $x=a_{1} \cdot v_{1}+a_{2}$. $v_{2} \cdots a_{n} \cdot v_{n}$ where each $a_{i} \in F$. We define the euclidean norm of $F^{n}$ corresponding to the basis $\left\{v_{i}\right\}$ in the following way, $\|x\|_{F^{n}}=\sqrt{a_{1}^{2}+a_{2}^{2}+\cdots+a_{n}^{2}}$.

Exercise: Show that any two euclidean norms on $F^{n}$ are equivalent.

Definition 5.3 (Subeuclidean norm). Let $F$ be either $\mathbb{Z}$ or a subfield of $\mathbb{C}$. Let $G$ be a semidirect product of $F^{n}$ by $\mathbb{Z}$. We will call the product norm on $G$ induced by the canonical word norm on $\mathbb{Z}$ and by a euclidean norm on $F$ a subeuclidean norm on G.

Observation 5.4. By Corollary A.4, if $F$ is $\mathbb{Z}$ or a subfield of $\mathbb{C}$ and $\phi \in \operatorname{GL}(n, F)$ then any two subeuclidean norms on $F^{n} \rtimes_{\phi} \mathbb{Z}$ are equivalent.

Lemma 5.5. Let $F$ be either $\mathbb{Z}$ or a subfield of $\mathbb{C}$. Let $G$ be a semidirect product of $F^{n}$ by $\mathbb{Z}$. Suppose $G$ is equipped with a subeuclidean norm. Then:

1. If $f \in F^{n}$ then $\|($ af $)\|\leq \max \{|a|, 1\} \cdot\| f \|, \forall a \in F$

2. If $f \in F^{n}$ then $\tau(a f)=|a| \cdot \tau(f), \forall a \in F$

Proof. Let $f \in F^{n}, a \in F-\{0\}$. Let $w=t^{n_{1}} f_{1} t^{n_{2}} f_{2} \cdots t^{n_{j}} f_{j}$ represent $f \in G$. Then by Lemma A.6, $w^{\prime}=t^{n_{1}} a f_{1} t^{n_{2}} a f_{2} \cdots t^{n_{j}} a f_{j}$ represents $a f \in G$. Now,

$$
\begin{aligned}
l\left(w^{\prime}\right)_{G} & =\sum_{i=1}^{n}\left|n_{i}\right|+\left\|a f_{i}\right\|_{F^{n}}=\sum_{i=1}^{n}\left|n_{i}\right|+|a|\left\|f_{i}\right\|_{F^{n}} \\
& \leq \max \{|a|, 1\} \cdot\left(\sum_{i=1}^{n}\left|n_{i}\right|+\left\|f_{i}\right\|_{F^{n}}\right)=\max \{|a|, 1\} \cdot l(w)_{G}
\end{aligned}
$$


Thus $\|a f\|_{G} \leq \max \{|a|, 1\} \cdot\|f\|_{G}$. Choose $m \in \mathbb{N}$ such that $|m a| \geq 1$, and apply Theorem 4.5 to get

$$
\begin{aligned}
\left.m \cdot \tau(a f)_{G}\right) & =\tau(\text { maf })_{G}=\lim _{n \rightarrow \infty} \frac{\|n m a f\|}{n} \leq \lim _{n \rightarrow \infty} \frac{\max \{|m a|, 1\} \cdot\|(n f)\|}{n} \\
& =\max \{|m a|, 1\} \cdot \tau(f)_{G}=m \cdot|a| \cdot \tau(f)_{G} .
\end{aligned}
$$

Hence, canceling $m$ 's, we get $\tau(a f) \leq|a| \cdot \tau(f)$. Since $a$ was arbitrary, we finally have $\tau(a f) \leq|a| \cdot \tau(f) \leq|a| \cdot|1 / a| \cdot \tau(a f)=\tau(a f)$.

Lemma 5.6. Let $\phi \in \operatorname{GL}(n, \mathbb{Z})$. Let $G=\mathbb{Z}^{n} \rtimes_{\phi} \mathbb{Z}$. Let $K=\mathbb{Q}^{n} \rtimes_{\phi} \mathbb{Z}$. By choosing a free basis for $\mathbb{Z}^{n}$ and using this as a vector space basis for $\mathbb{Q}^{n}$, we may consider $G<K$. Under these circumstances the subeuclidean norm on $G$ and the norm induced by restricting the subeuclidean norm on $K$ to $G$ induce translation functions that are equal on $\mathbb{Z}^{n}$.

Proof. Let us call the respective norms \|\|$_{G}$ and \|\|$_{K}$ and their translation functions $\tau_{G}$ and $\tau_{K}$. It is clear from the definition of the norms that \|\|$_{G} \geq\|\|_{K}$ and thus $\tau_{G} \geq \tau_{K}$. Let $z \in \mathbb{Z}^{n}$. Now, from the definition of the product metric, for each $i \in \mathbb{N}$ there is a string $w_{i}=t^{n_{1}} q_{1 i} \cdots t^{n_{j}} q_{j i}$ representing $z^{i}$ in $K$, where $t$ is a generator of $\mathbb{Z}, q_{m i} \in \mathbb{Q}^{n}$ and $l\left(w_{i}\right)_{K} \leq\left\|z^{i}\right\|_{K}+2^{-i}$ (where $l\left(w_{i}\right)_{K}$ is as defined in the definition of product metric). Let $r_{i}$ be the least common multiple of the entries of the $q_{m i}$ 's. By Lemma A.6, $z^{r_{i} \cdot i}=t^{n_{1}}\left(q_{1 i} \cdot r_{i}\right) \cdots t^{n_{j}}\left(q_{j i} \cdot r_{i}\right)$ (using additive notation in the abelian group $\mathbb{Q}$ and multiplicative notation in $K$ ), where $\left(q_{m i} \cdot r_{i}\right)$ is in $\mathbb{Z}^{n}$. Hence, $\left\|z^{r_{i} \cdot i}\right\|_{G} \leq r_{i} \cdot l\left(w_{i}\right)_{K} \leq r_{i} \cdot\left(\left\|z^{i}\right\|_{K}+2^{-i}\right)$. Thus, $\tau(z)_{G} \leq \frac{\left\|z^{r_{i} \cdot i}\right\|_{G}}{r_{i} \cdot i} \leq \frac{r_{i} \cdot\left(\left\|z^{i}\right\|_{K}+2^{-i}\right)}{r_{i} \cdot i}=\frac{\left\|z^{i}\right\|_{K}+2^{-i}}{i}$. Finally, letting $i$ tend to infinity, we see that $\tau(z)_{G} \leq \tau(z)_{K}$.

Corollary 5.7. Let $\phi \in \operatorname{GL}(n, \mathbb{Z})$. A word norm on $\mathbb{Z}^{n} \rtimes_{\phi} \mathbb{Z}$ and a norm on $\mathbb{Z}^{n} \rtimes_{\phi} \mathbb{Z}$ induced by restricting any subeuclidean metric on $\mathbb{C}^{n} \rtimes_{\phi} \mathbb{Z}$ have translation functions that are equivalent when restricted to $\mathbb{Z}^{n}$.

Proof. By Corollary A.4 the product norms on $\mathbb{Z}^{n} \rtimes_{\phi} \mathbb{Z}$ induced by a word norm and a subeuclidean norm on $\mathbb{Z}^{n}$ are equivalent and thus induce equivalent translation functions. The previous lemma tells us that the subeuclidean norms on $\mathbb{Z}^{n} \rtimes \mathbb{Z}$ and $\mathbb{Q}^{n} \rtimes \mathbb{Z}$ induce equal translation functions on $\mathbb{Z}^{n}$. lemma A.7 implies that the subeuclidean norms on $\mathbb{Q}^{n} \rtimes \mathbb{Z}$ and $\mathbb{R}^{n} \rtimes \mathbb{Z}$ agree on $\mathbb{Q}^{n}$. Similarly, Lemma A.8 implies that the subeuclidean norms on $\mathbb{R}^{n} \rtimes \mathbb{Z}$ and $\mathbb{C}^{n} \rtimes \mathbb{Z}$ agree on $\mathbb{R}^{n}$.

Definition 5.8 (Subspace of $\mathbb{Z}^{n}$ ). We say that a subgroup $H$ of $Z^{n}$ is a subspace of $\mathbb{Z}^{n}$ of dimension $d$ if there exists an embedding of $\mathbb{Z}^{n}$ into $\mathbb{Q}^{n}$ so that $H$ is the intersection of $\mathbb{Z}^{n}$ with a $d$-dimension vector subspace of $\mathbb{Q}^{n}$. Equivalently, $H$ is a subspace of dimension $d$ if $\sqrt{H}=H$ and $H$ has rank $d$.

Observation 5.9. The following facts will be used throughout the arguments in this section.

1. If $T$ is a subspace of $\mathbb{Z}^{n}$ of dimension $d$ then $\mathbb{Z}^{n} / T \simeq \mathbb{Z}^{n-d}$.

2. The intersection of subspaces is a subspace.

3. The image of a subspace under an element of $\operatorname{GL}(n, \mathbb{Z})$ is a subspace.

Definition 5.10 (Invariant Subspace). Let $\phi \in \mathrm{GL}(n, \mathbb{Z}), T$ a subspace of $\mathbb{Z}^{n}$. We say that $T$ is invariant under $\phi$ or ( $T$ is $\phi$-invariant) if $\phi(T) \subseteq T$. 
Lemma 5.11. Let $\phi \in \mathrm{GL}(n, \mathbb{Z}), T$ a subspace of $\mathbb{Z}^{n}$. Then $T$ is invariant under $\phi$ if and only if $\phi(T)=T$.

Proof. $\phi(T) \subseteq T \Rightarrow T \subseteq \phi^{-1}(T)$, also $T$ and $\phi^{-1}(T)$ have the same rank. Since $T$ is a subspace, $T=\phi^{-1}(T)$ and thus $\phi(T)=T$.

Lemma 5.12. Let $\mathbb{F}$ be either $\mathbb{Z}$ or a the additive group of a subfield of $\mathbb{C}$. Let $G$ be a semidirect product of the form $\mathbb{F}^{n} \rtimes_{\phi} \mathbb{Z}$, where $\phi \in \operatorname{GL}(n, \mathbb{F})$. If $G$ is equipped with a subeuclidean norm then $I(G)$ is a $\phi$-invariant subspace of $\mathbb{F}^{n}$.

Proof. Now $G / \mathbb{F}^{n}=\mathbb{Z}$ and since $G$ was equipped with a subeuclidean norm, the induced metric on $\mathbb{Z}$ is the canonical one. Thus $I(\mathbb{Z})=1$. Applying Observation 4.8 we see that if $\gamma: G \longrightarrow \mathbb{Z}$ is the natural map, then $\tau_{G}(g) \geq \tau_{\mathbb{Z}}(\gamma(g)) \forall g \in G$. Thus $\gamma(I(G))=\{1\}$ and so $I(G) \leq \mathbb{F}^{n}$. Let $g, h \in I(G), a, b \in \mathbb{F}$. $\tau(a g+b h) \leq$ $\tau(a g)+\tau(b h)$ since $\mathbb{F}^{n}$ is abelian. Thus applying Lemma $5.5 \tau(a g+b h)=|a|$. $\tau(g)+|b| \cdot \tau(h)=0+0=0$. Thus $I(G)$ is a subgroup of $\mathbb{F}^{n}$. It is also evident that $\sqrt{I(G)}=I(G)$ and thus by the equivalent definition at the end of definition 5.8, $I(G)$ is a subspace of $\mathbb{F}^{n}$. $I(G)$ is $\phi$-invariant since

$$
\tau(\phi(a))=\tau\left(t^{-1} a t\right)=\tau(a)=0
$$

for every $a$ in $I(G)$, where $t$ is the generator for $\mathbb{Z}$.

Lemma 5.13. Let $\phi \in \mathrm{GL}(n, \mathbb{Z})$. Suppose $T$ is a nontrivial subspace of $\mathbb{Z}^{n}$ invariant under $\phi$ of dimension $d$, Then $\left.\phi\right|_{T}$ acts as an element of $\operatorname{GL}(d, \mathbb{Z})$ with a monic minimal polynomial of degree $\leq d$. Conversely, if $f$ is a polynomial of degree $k$ that divides the minimal polynomial of $\phi$ then there is a $\phi$-invariant subspace $S_{f}$ of $\mathbb{Z}^{n}$ of dimension $\geq k$ such that $f$ is the minimal polynomial of $\left.\phi\right|_{S_{f}}$.

Proof. The first half is obvious. For the second half, embed $\mathbb{Z}^{n}$ in $\mathbb{Q}^{n}$ and let $S_{f}=\operatorname{ker}_{\mathbb{Q}^{n}}(f(\phi)) \cap \mathbb{Z}^{n}$.

Definition 5.14 (Irreducible). We call an element of $\operatorname{GL}(n, \mathbb{Z})$ irreducible if it has no proper nontrivial invariant subspaces in $\mathbb{Z}^{n}$, or equivalently if its minimal polynomial is irreducible over $\mathbb{Z}$.

Lemma 5.15. Let $\phi \in \mathrm{GL}(n, \mathbb{Z})$. Let $f$ be the characteristic polynomial of $\phi$. Write $f=f_{1} \cdot f_{2} \cdots f_{t}$ where each $f_{t}$ is a power of a different monic prime polynomial over $\mathbb{Z}$. Then there exists $\left\{T_{1}, \cdots, T_{t}\right\}$, a set of $\phi$-invariant subspaces of $\mathbb{Z}^{n}$, such that the characteristic polynomial of $\left.\phi\right|_{T_{i}}$ is $f_{i}, T_{i} \cap T_{j}=0$ if $i \neq j$, and the direct sum of the $\left\{T_{i}\right\}$ has finite index in $\mathbb{Z}^{n}$.

Proof. Embed $\mathbb{Z}^{n}$ in $\mathbb{Q}^{n}$. Now $\mathbb{Q}^{n}$ can be written as $\mathbb{Q}^{n}=\bigoplus_{i=1}^{t} V_{i}$ where the characteristic polynomial of $\left.\phi\right|_{V_{i}}$ is $f_{i}$. Let $T_{i}=V_{i} \cap \mathbb{Z}^{n}$. So, the direct sum of the $\left\{T_{i}\right\}$ is a free abelian subgroup of $\mathbb{Z}^{n}$ of rank $n$, and thus has finite index in $\mathbb{Z}^{n}$.

Lemma 5.16. Let $\phi \in \operatorname{GL}(n, \mathbb{Z})$ be irreducible. Equip $G=\mathbb{C}^{n} \rtimes_{\phi} \mathbb{Z}$ with a subeuclidean norm induced by a basis of eigenvectors of $\phi$. Let $v \in \mathbb{C}^{n}$ be an eigenvector of $\phi$. Then $\tau_{G}(v)$ is nonzero if and only if the eigenvalue of $v$ has complex norm 1 , in which case $\tau_{G}(v)=\|v\|_{\mathbb{C}^{n}}=\|v\|_{G}$.

Proof. Let $\lambda$ be the eigenvalue corresponding to $v$. If $\|\lambda\| \neq 1$, choose $t$ a generator of $\mathbb{Z}$ and $\beta \in\{\lambda, 1 / \lambda\}$ such that $\|\beta\|<1$ and $t^{-1} v t=\beta v$. Then $\tau(v)=\tau\left(t^{-n} v t^{n}\right) \leq$ $\left\|t^{-n} v t^{n}\right\| \leq\left\|\beta^{n}\right\|\|v\| \rightarrow 0$ as $n \rightarrow \infty$. Suppose $\|\lambda\|=1$. Since $f$ the minimal 
polynomial of $\phi$ is irreducible over $\mathbb{Z}$, it has no repeated roots. Hence, not only is $\langle v\rangle$, the subspace of $\mathbb{C}^{n}$ spanned by $v$, invariant under $\phi$, but also its complementary space, $\langle v\rangle \perp$, is invariant under $\phi$ (one may see this by noting that, in this basis, $\phi$ is in Jordan normal form and has 1-by-1 Jordan blocks and so is diagonal). Thus, by Lemma A.8, while calculating the norms of elements of $\{n v \mid n \in \mathbb{N}\}$ we need consider only strings of the form $w_{n}=t^{n_{1}} \gamma_{1} v t^{n_{2}} \gamma_{2} v \cdots t^{n_{j}} \gamma_{j} v, \gamma_{i} \in \mathbb{C}$ which represent $n v$. Replacing $w_{n}$ by a product of elements in $\langle v\rangle$ conjugated by powers of $t$ as in the proof of Lemma A.5 and recalling that $t^{-i} v t^{i}=\lambda v$ we see that we have $w_{n}^{\prime}=t^{n_{1}} \gamma_{1} v t^{-n_{1}} t^{n_{1}+n_{2}} \gamma_{2} v \cdots \gamma_{j} v$ which we can write as $w_{n}^{\prime \prime}=$ $\lambda^{n_{1}} \gamma_{1} v \lambda^{n_{1}+n_{2}} \gamma_{2} v \cdots \gamma_{j} v$ and $l\left(w^{\prime \prime}\right)_{G} \leq l(w)_{G}$ since $|\lambda|=1$. So, while calculating the norms of elements of $\{n v\}$ we need consider only the metric on $\mathbb{C}^{n}$. Hence, $\tau_{G}(v)=\tau_{\mathbb{C}^{n}}(v)=\|v\|_{\mathbb{C}^{n}}=\|v\|_{G}$.

Lemma 5.17. Let $\left(\lambda_{1}, \lambda_{2}, \cdots, \lambda_{n}\right)$ be an $n$-tuple of elements in $\mathbb{C}^{n}$ such that $\left|\lambda_{i}\right|=$ 1 for each $i$. Then for any $\epsilon>0$ there exist $M, N \in \mathbb{N}, M \neq N$ such that $\mid \lambda_{i}^{M}-$ $\lambda_{i}^{N} \mid<\epsilon$ for all $i$.

Proof. Identify $S^{1}$ with the set $\{z \in \mathbb{C}|| z \mid=1\}$. Then each n-tuple of the form $\left(\lambda_{1}^{i}, \lambda_{2}^{i}, \cdots, \lambda_{n}^{i}\right), i \in \mathbb{N}$ lies on the $\mathrm{n}$-dimensional torus, $T^{n}$. The result follows from the compactness of $T^{n}$.

Lemma 5.18. Let $\phi \in \mathrm{GL}(n, \mathbb{Z})$ be irreducible. Let $G=\mathbb{Z}^{n} \rtimes_{\phi} \mathbb{Z}$. Then $G$ is translation discrete implies that $\phi$ has finite order and thus $G$ is abelian-by-finite.

Proof. Choose a basis $\left\{a_{i}\right\}$ for $\mathbb{Z}^{n}$ and $t$ a generator for $\mathbb{Z}$. By Lemma 2.4, we may assume that $G$ is equipped with the associated word metric. In light of Corollary 5.7 we may consider $G$ as a subgroup of $K=\mathbb{C}^{n} \rtimes_{\phi} \mathbb{Z}$ (K is equipped, as usual, with the subeuclidean norm) and calculate translation numbers there. Since $\phi$ is irreducible, $f$, the minimal polynomial of $\phi$, is irreducible over $\mathbb{Z}$. Since $f$ is irreducible, each root has multiplicity 1 . This, in turn, implies that $\phi$ is diagonalizable (since when $\phi$ is written in Jordan normal form the Jordan blocks will be 1-by-1). Thus, we may choose as a basis for $\mathbb{C}^{n}$ a set of eigenvectors of $\phi$. By Corollary 5.7, we may assume that $K$ is equipped with subeuclidean norm induced by the subeuclidean norm on $\mathbb{C}^{n}$ induced by this basis. Let $\left\{\lambda_{i}\right\},(i=1 \ldots r)$ be the set of eigenvalues of $\phi$ with $\left|\lambda_{i}\right| \neq 1$, and $v_{i}$ be the basis vector corresponding to $\lambda_{i}$. Similarly, let $\left\{\gamma_{j}\right\},(j=1 \ldots s)$ be the set of eigenvalues of $\phi$ with $\left|\gamma_{j}\right|=1$, and $w_{j}$ be the basis vector corresponding to $\gamma_{j}$. Let $g \in \mathbb{C}^{n}$ be an element of the image of $\mathbb{Z}^{n}$. In the new basis, $g$ can be written as $g=a_{1} v_{1}+a_{2} v_{2}+\cdots+a_{r} v_{r}+b_{1} w_{1}+\cdots+b_{s} w_{s}, a_{i}, b_{j} \in \mathbb{C}$. Choose $\epsilon>0$ such that $\tau(h)<\epsilon$ implies that $h=1_{G}$, for all $h \in G$. Let $B=\max \left\{b_{j}\right\}$. Apply the previous lemma to find $n, m \in \mathbb{N}, m \neq n$ such that $\left|\gamma_{j}^{n}-\gamma_{j}^{m}\right|<\frac{\epsilon}{s \cdot B}$ for $j=1 \cdots s$. Consider $g^{\prime}=t^{-n} g t^{n} t^{-m} g^{-1} t^{m}$. Then $g^{\prime}=$ $\left(\lambda_{1}^{n}-\lambda_{1}^{m}\right) a_{1} v_{1}+\cdots+\left(\lambda_{r}^{n}-\lambda_{r}^{m}\right) a_{r} v_{r}+\left(\gamma_{1}^{n}-\gamma_{1}^{m}\right) b_{1} w_{1}+\cdots+\left(\gamma_{s}^{n}-\gamma_{s}^{m}\right) b_{s} w_{s}$. Applying, Lemma 5.16, Lemma 5.12, and Corollary 4.6 we have that

$$
\begin{aligned}
\tau\left(g^{\prime}\right) & \leq \tau\left(\left(\gamma_{1}^{n}-\gamma_{1}^{m}\right) b_{1} w_{1}+\cdots+\left(\gamma_{s}^{n}-\gamma_{s}^{m}\right) b_{s} w_{s}\right) \\
& \leq\left\|\left(\gamma_{1}^{n}-\gamma_{1}^{m}\right) b_{1} w_{1}+\cdots+\left(\gamma_{s}^{n}-\gamma_{s}^{m}\right) b_{s} w_{s}\right\|_{G} \\
& \leq\left\|\left(\gamma_{1}^{n}-\gamma_{1}^{m}\right) b_{1} w_{1}+\cdots+\left(\gamma_{s}^{n}-\gamma_{s}^{m}\right) b_{s} w_{s}\right\|_{\mathbb{C}^{n}} \\
& \leq\left|\left(\gamma_{1}^{n}-\gamma_{1}^{m}\right) b_{1}\right|+\cdots+\left|\left(\gamma_{s}^{n}-\gamma_{s}^{m}\right) b_{s}\right| \\
& \leq s \cdot \frac{\epsilon}{s \cdot B} \cdot B \\
& =\epsilon .
\end{aligned}
$$


Thus, $g^{\prime}=1_{G}$. So, $t^{-n} g t^{n} t^{-m} g^{-1} t^{m}=1$ and thus $\left[t^{m-n}, g\right]=1$. Hence, letting $g$ run through the images of a free basis for $\mathbb{Z}^{n}$, we see that $\phi$ has finite order proving that $G$ is abelian-by-finite.

Theorem 5.19. Let $\phi \in \operatorname{GL}(n, \mathbb{Z})$. Let $G=\mathbb{Z}^{n} \rtimes_{\phi} \mathbb{Z}$. Then $G$ is translation discrete if and only if $G$ is abelian-by-finite.

Proof. If $G$ is translation discrete, then any subgroup of $G$ is translation discrete. Applying the previous lemma we see that(in the notation of Lemma 5.15) $T_{i} \rtimes_{\phi \mid T_{i}} \mathbb{Z}$ is abelian-by-finite, forcing $\phi \mid T_{i}$ to have finite order. Since the direct sum of the $T_{i}$ 's has finite index in $\mathbb{Z}^{n}$, this forces $\phi$ to have finite order, which implies that $G$ is abelian-by-finite.

\section{Solvable Groups}

We need a lemma from a previous article.

Corollary 6.1 ([C2], Cor. 3.6.1). Translation proper nilpotent groups are abelian.

Proof of Theorem 3.3. Let $G$ be a translation definite solvable linear group. It has been shown by Mal'cev [S, Corollary 4, Theorem 4 page 35] that a solvable linear group is (nilpotent-by-abelian)-by-finite. Since the property of being translation proper is inherited by subgroups we may apply Corollary 6.1 to get that $G$ is metabelian-by-finite.

Lemma 6.2. Let $G$ be a solvable group equipped with a metric so that $\tau^{-1}(0)=1_{G}$. Let $A$ be a maximal abelian normal subgroup of $G$. Then $C_{G}(A)=A$ where $C_{G}(A)$ is the centralizer of $A$ in $G,\left\{g \in G \mid[g, A]=1_{G}\right\}$.

Proof. Let $B / A$ be a maximal abelian characteristic subgroup of the solvable group $C_{G}(A) / A$. Since $C_{G}(A) / A$ is normal in $G / A$, and $B / A$ is characteristic in $C_{G}(A) / A$, $B / A$ is normal in $G / A$. Thus $B$ is a normal subgroup of $G$. Since $B / A$ is abelian, $B^{\prime}=[B, B]$ is generated by commutators in $A$ and thus in the center of $B$. In proof of [C2, Lemma 3.2.1] it is shown that central commutators have zero translation number in any metric (in that lemma it is assumed that the group is nilpotent, but this hypothesis is used only to force commutators to lie in the center).

Thus, $\tau\left(B^{\prime}\right)=0$. So, $B^{\prime}=1_{G}$. Therefore $B$ is an abelian normal subgroup of $\mathrm{G}$ which contains $A$. Thus $B=A$. Then, $B / A=1_{G} \Rightarrow C_{G}(A) / A=1_{G} \Rightarrow C_{G}(A)=$ A.

Definition 6.3 (Rank). If $A$ is an abelian group, then the rank of $A$ is defined as:

$$
\operatorname{rank}(A)=\operatorname{dim}_{\mathbb{Q}}\left(A \otimes_{\mathbb{Z}} \mathbb{Q}\right) .
$$

This is also known as the torsion-free rank or $\mathbb{Q}$-rank.

Lemma 6.4. Let $G$ be a solvable group which is translation proper. Let $A$ be a maximal abelian normal subgroup of $G$. If $A$ has finite $\mathbb{Q}$-rank and is torsion-free then $G$ is metabelian-by-finite (i.e. $G$ is abelian-by-abelian-by-finite).

Proof. Since $A$ torsion-free, $A$ embeds into $A \otimes \mathbb{Q}$ by $a \mapsto a \otimes 1$. Since $A$ has finite rank, $A \leq A \otimes \mathbb{Q} \leq \mathbb{Q}^{n}$ for some $n$. By Lemma $6.2, G / A=G / C_{G}(A)$, so $G / A$ is a subgroup of $\operatorname{Aut}\left(\mathbb{Q}^{n}\right)=\operatorname{GL}(n, \mathbb{Q})$. Since $G / A$ is linear, a theorem in $([\mathrm{S}])$ allows us to choose a torsion-free subgroup of finite index, $K / A$. Applying another theorem in $([\mathrm{S}])$, there is a basis of $\mathbb{C}^{n}$ in which $K / A$ is a group of upper triangular 
matrices. Now any commutator $\phi$ in $K / A$ is unipotent (since it is the commutator of two upper triangular matrices), implying that $\langle\phi, A\rangle=A \rtimes_{\phi} \mathbb{Z}$ is nilpotent and thus abelian by Corollary 6.1 which shows that $\phi$ is the identity. So $K / A$ is abelian implying that $G$ is metabelian-by-finite.

Proof of Theorem 3.1. A solvable subgroup of a group with finite virtual cohomological dimension itself has finite vcd. Thus it is torsion-free and does not contain a free abelian group of infinite rank and consequently has finite $\mathbb{Q}$-rank. We may now apply Lemma 6.4 to complete the proof.

Lemma 6.5. Let $G$ be torsion-free solvable translation discrete group. Let $A$ be a maximal abelian normal subgroup of $G$. If $A$ has finite $\mathbb{Q}$-rank $m$, then $G$ is a finite extension of $A \cong \mathbb{Z}^{m}$.

Proof. Since $A$ is a subgroup of $G$ it is itself translation discrete, and thus can have no infinitely divisible elements by Theorem 4.5. Hence $A$ is isomorphic to $\mathbb{Z}^{m}$. Let $x \in G$. Then $\langle x, A\rangle$ is, by Theorem 5.19, abelian-by-finite. Thus, there is a finite power, $t$, of $x$ so that $x^{t} \in C_{G}(A)$. By Lemma 6.2 , we have $C_{G}(A)=A$. This implies that $G / A$ is a torsion group. Also, $G / A=G / C_{G}(A)$ is a subgroup of $\operatorname{Aut}(A)=\mathrm{GL}(m, \mathbb{Z})$. By $[\mathrm{S}$, Cor 1,page 26$]$, Every solvable subgroup of $\mathrm{GL}(m, \mathbb{Z})$ is polycyclic. Therefore, $G / A$ is a polycyclic torsion group and thus is finite.

Proof of Theorem 3.4. By descending to a subgroup of finite index and applying Lemma 2.4 we may assume that we are considering a solvable translation discrete group $H$ of finite cohomological dimension $n$. Now, $H$ is torsion-free and, by Zorn's Lemma, has a non-trivial maximal abelian normal subgroup $A$. Since the cohomological dimension of $A$ is no greater than that of $H, m$, the $\mathbb{Q}$-rank of $A$, is no greater than $n$. Applying Lemma 6.5 we see that $H$ must be a finite extension of $\mathbb{Z}^{m}$. But since $A$ now has finite index in $H, m=n$.

\section{Two Examples}

Example 7.1. Let $\phi$ be the matrix

$$
\left(\begin{array}{rrrr}
0 & 0 & 0 & -1 \\
1 & 0 & 0 & 2 \\
0 & 1 & 0 & -1 \\
0 & 0 & 1 & 2
\end{array}\right) .
$$

Let $G=\mathbb{Z}^{4} \rtimes_{\phi} \mathbb{Z}$. Choose a finite generating set for $G$ and equip $G$ with the corresponding word metric. As we shall show below, $\tau^{-1}(0)=\left\{1_{G}\right\}$, but the set $S=\left\{\tau(g) \mid g \in \mathbb{Z}^{n}\right\}$ has the property that every point of $S$ is an accumulation point of $S$. Thus $G$ is translation proper, but not translation discrete.

It is an easy calculation that $f(x)=x^{4}-2 x^{3}+x^{2}-2 x+1$, the characteristic polynomial of $\phi$, is irreducible over $\mathbb{Z}$, and has a root, $\alpha=\frac{1-\sqrt{2}}{2}+i \cdot \frac{\sqrt{1+2 \sqrt{2}}}{2}$, of complex norm 1 which is not a root of unity. Since $f$ is irreducible it is, in fact, the minimal polynomial of $\phi$. Thus $\phi$ is irreducible. Also, $T=I(G)$ is an invariant subspace of $\mathbb{Z}^{n}$. Hence, either $T=\mathbb{Z}^{n}$ or $T=\{0\}$. Suppose $T=\mathbb{Z}^{n}$. Let $K=\mathbb{C}^{n} \rtimes_{\phi} \mathbb{Z}$ be equipped a subeuclidean metric. By Corollary 5.7, the image of $\mathbb{Z}^{n}$ is contained inside $I(K)$, but the image of $\mathbb{Z}^{n}$ contains a basis of $\mathbb{C}^{n}$. Since $I(K)$ is a vector subspace of $\mathbb{C}^{n}, I(K)=\mathbb{C}^{n}$. On the other hand, let $v$ be an eigenvector of $\phi$ in $\mathbb{C}^{n}$ corresponding to the eigenvalue $\alpha$. Then, by Lemma 5.16, 
$\tau_{K}(v)=\|v\|_{K} \neq 0$, which gives us a contradiction. Thus $I(G)=T=\{0\}=\left\{1_{G}\right\}$. However, since $\alpha$ is not a root of unity, $\phi$ does not have finite multiplicative order, and thus $G$ is not abelian-by-finite. It follows from Theorem 5.19 that $G$ is not translation discrete.

If $x \in G-\mathbb{Z}^{n}$ we can apply Observation 4.8 to show that $\tau(x)$ is bounded away from 0 . Thus 0 must be an accumulation point of $S$. Since $\mathbb{Z}^{n}$ is abelian, for any elements $x, y \in \mathbb{Z}^{n}$ we have (by Theorem 4.5) $|\tau(x)-\tau(y)| \leq \tau(x y) \leq \tau(x)+\tau(y)$. For $\tau(y) \neq 0$ and for sufficiently large $n$ we have that $\tau(x)<\left|\tau\left(y^{n}\right)-\tau(x)\right| \leq \tau\left(x y^{n}\right)$. Choosing $n \in \mathbb{N}$ minimal with this property one calculates that $\tau(x)<\tau\left(x y^{n}\right) \leq$ $\tau\left(x y^{n-1}\right)+\tau(y) \leq \tau(x)+\tau(y)$. Choosing $\tau(y)$ increasingly small, we see that $\tau(x)$ is an accumulation point of $S$ for any element $x$ of $\mathbb{Z}^{n}$.

Example 7.2. Let $\phi$ be the automorphism of the free abelian group of countably infinite rank induced by shifting a free basis indexed by $\mathbb{Z}$ to the right. Let $G$ be the induced HNN extension ( $G$ is also known as a wreath product of two copies of $\mathbb{Z}$ ). Then $G$ is generated by two elements (t, the extra generator from the HNN extension, and x, one of the generators of one of the $\mathbb{Z}$ 's, will work) and $G$ is translation discrete.

Let $x_{i}=t^{-i} x t^{i}$. Then the set $\left\{x_{i}\right\}$ forms a free basis for $H$. By Observation 4.8, we need consider only elements of $H$. So, each element, $h \neq 0$, of $H$ can be written uniquely in the form $h=a_{1} x_{i_{1}}+a_{2} x_{i_{2}}+\cdots+a_{k} x_{i_{k}}, a_{i} \in \mathbb{Z}-0$. One shows that in the word metric on $G$ induced by the generating set $\{t, x\},\|h\| \geq \sum\left|a_{i}\right|$. Then

$$
\tau(h)=\lim _{n \rightarrow \infty} \frac{\|n h\|}{n} \geq \lim _{n \rightarrow \infty} \frac{\sum\left|n a_{i}\right|}{n}=\sum\left|a_{i}\right| \geq 1 .
$$

\section{ApPendix A. ElEMENTARY RESUlts on GROUP MEtRICS}

The following results are quite useful when working with metrics on abstract groups. Proofs are given only for the those results which are not straightforward.

Lemma A.1. Suppose the finitely generated group $G$ is a semidirect product of $K$ by the finitely generated group $H$. Suppose $s$ is a finite generating set for $H$ and $r \supseteq s$ is one for $G$. Suppose we equip $G$ and $H$ with the corresponding word metrics, then $\left.\tau_{G}\right|_{H}=\tau_{H}$.

Lemma A.2. Suppose $H$ and $K$ are subgroups of $G$ that together generate $G$, each is equipped with a metric, and $G$ is equipped with the product semimetric. If the metric on $H$ is discrete, then the product semimetric on $G$ is a metric and there exists $\epsilon>0$ such that for all $g \in G,\|g\|_{G}<\epsilon \Rightarrow g \in K$ and $\|g\|_{G}=\|g\|_{K}$.

Lemma A.3. Suppose $H$ and $K$ are subgroups of $G$ that together generate $G$. Suppose $K$ is equipped with a discrete metric, and $H$ is equipped with two semimetrics \|\|$_{H_{1}} \preceq\|\|_{H_{1}}$ (in the terms of the previously defined partial order). Then the two product semimetrics on $G$ have the property that \|\|$_{G_{1}} \preceq\|\|_{G_{2}}$.

Corollary A.4. Suppose $H$ and $K$ are subgroups of $G$ that together generate $G$. Suppose $K$ is equipped with a discrete metric, and $H$ is equipped with two equivalent semimetrics. Then the two product semimetrics on $G$ are equivalent.

Lemma A.5. Let $K$ be an abelian group, $G$ a semidirect product of $K$ by $\mathbb{Z}$, and $t$ a generator of $\mathbb{Z}$. If $w=\left(t^{n_{1}} g_{1} t^{n_{2}} g_{2} \cdots t^{n_{j}} g_{j}\right)\left(t^{n_{1}} h_{1} t^{n_{2}} h_{2} \cdots t^{n_{j}} h_{h}\right)\left(g_{i}, h_{i} \in K\right)$ is an element of $G$ that lies in $K$, then $w=t^{n_{1}} g_{1} h_{1} t^{n_{2}} g_{2} h_{2} \cdots t^{n_{j}} g_{j} h_{j}$. 
Proof. Since $w$ lies in $K, \sum_{i=1}^{j} n_{i}=0$.

$$
\begin{gathered}
w=\left(t^{n_{1}} g_{1} t^{-n_{1}} t^{n_{1}+n_{2}} g_{2} t^{-n_{1}-n_{2}} \cdots t^{n_{1}+n_{2}+\cdots+n_{j-1}} g_{j-1} t^{-n_{1}-n_{2}-\cdots-n_{j-1}} g_{j}\right) \\
\left(t^{n_{1}} h_{1} t^{-n_{1}} t^{n_{1}+n_{2}} h_{2} t^{-n_{1}-n_{2}} \cdots t^{n_{1}+n_{2}+\cdots+n_{j-1}} h_{j-1} t^{-n_{1}-n_{2}-\cdots-n_{j-1}} h_{j}\right) .
\end{gathered}
$$

Since $K$ is abelian and normal any two conjugates of elements of $K$ commute and we have $w=t^{n_{1}} g_{1} h_{1} t^{-n_{1}} t^{n_{1}+n_{2}} g_{2} h_{2} t^{-n_{1}-n_{2}} \cdots t^{n_{1}+\cdots+n_{j-1}} g_{j-1} h_{j-1} t^{-n_{1}-\cdots-n_{j-1}} g_{j} h_{j}$. Canceling $t$ 's we get $w=t^{n_{1}} g_{1} h_{1} t^{n_{2}} g_{2} h_{2} \cdots t^{n_{j}} g_{j} h_{j}$.

Lemma A.6. Let $F$ be a subring of $\mathbb{C}, \phi \in \mathrm{GL}(n, F), G=F^{n} \rtimes_{\phi} \mathbb{Z}$. Let $t$ denote a generator of $\mathbb{Z}$. If $w=\left(t^{n_{1}} g_{1} t^{n_{2}} g_{2} \cdots t^{n_{j}} g_{j}\right)\left(g_{i} \in G\right)$ is an element of $G$ which lies in $F^{n}$, then $a w=\left(t^{n_{1}} a g_{1} t^{n_{2}} a g_{2} \cdots t^{n_{j}} a g_{j}\right) \forall a \in F$

Proof. Using multiplicative notation in $G$ and additive notation in $F^{n}$, $a w=a \cdot\left(t^{n_{1}} g_{1} t^{-n_{1}}+t^{n_{1}+n_{2}} g_{2} t^{-n_{1}-n_{2}}+\cdots+t^{n_{1}+\cdots+n_{j-1}} g_{j-1} t^{-n_{1}-\cdots-n_{j-1}}+g_{j}\right)$ $=\left(a \cdot t^{n_{1}} g_{1} t^{-n_{1}}+a \cdot t^{n_{1}+n_{2}} g_{2} t^{-n_{1}-n_{2}}+\cdots+a \cdot t^{n_{1}+\cdots+n_{j-1}} g_{j-1} t^{-n_{1}-\cdots-n_{j-1}}+a \cdot g_{j}\right)$ Since $\phi$ is a linear map we have $a w=\left(t^{n_{1}} a g_{1} t^{-n_{1}}+t^{n_{1}+n_{2}} a g_{2} t^{-n_{1}-n_{2}}+\cdots+t^{n_{1}+\cdots+n_{j-1}} a g_{j-1} t^{-n_{1}-\cdots-n_{j-1}}+a g_{j}\right)$ Canceling $t$ 's we get $a w=t^{n_{1}} a g_{1} t^{n_{2}} a g_{2} \cdots t^{n_{j}} a g_{j}$.

Lemma A.7. Suppose $G$ is an abelian group equipped with a semimetric and $H$ is a dense subgroup of $G$ with the restricted metric. Let $\phi$ be an automorphism of $G$ which is continuous and fixes $H$. Give $\mathbb{Z}$ a word metric induced by a generator. Let $K=H \rtimes_{\phi} \mathbb{Z}$ and $L=G \rtimes_{\phi} \mathbb{Z}$. Then the product metric on $K$ restricted to $H$ is equal to the product metric on $L$ restricted to $H$.

Proof. Let $h \in H, t$ be a generator of $\mathbb{Z}$. Suppose $w=t^{n_{1}} g_{1} t^{n_{2}} g_{2} \cdots t^{n_{j}} g_{j}, g_{i} \in G$ represents $h \in L$.Let $\epsilon>0$. Rewriting $w$, we get $w=t^{n_{1}} g_{1} t^{-n_{1}}+t^{n_{1}+n_{2}} g_{2} t^{-n_{1}-n_{2}}+$ $\cdots g_{j}$. Since $\phi$ is continuous and $H$ is dense we can find a sequence $\left(h_{1}, \ldots, h_{j}\right)$ of elements in $H$ such that $w^{\prime}=t^{n_{1}} h_{1} t^{n_{2}} h_{2} \cdots t^{n_{j}} h_{j}$ and $d\left(h, \overline{w^{\prime}}\right)<\epsilon / 2$ (where $\overline{w^{\prime}}$ is the element of $H$ which $w^{\prime}$ represents) and $l\left(w^{\prime}\right)_{K}<l(w)_{L}+\epsilon / 2$. Let $h^{\prime}=h-\overline{w^{\prime}}$. By construction $\left\|h^{\prime}\right\|_{H}<\epsilon / 2$. Hence, $w^{\prime \prime}=t^{n_{1}} h_{1} t^{n_{2}} h_{2} \cdots t^{n_{j}} h_{j} h^{\prime}$ has the property that it represents $h$ in $K$ and $l\left(w^{\prime \prime}\right)_{K}<l(w)_{L}+\epsilon$. Now, $\forall \epsilon>0$

$$
\begin{aligned}
\|h\|_{L} & =\inf \left\{l(w)_{L} \mid w \text { represents } h \text { in } L\right\} \\
& <\inf \left\{l\left(w^{\prime \prime}\right)_{K}+\epsilon \mid w^{\prime \prime} \text { represents } h \text { in } K\right\} \\
& =\|h\|_{K}+\epsilon
\end{aligned}
$$

Thus, $\|h\|_{L}=\|h\|_{K}$.

Lemma A.8. Suppose $G=H \times K$ is an abelian group equipped with a semimetric. Let $\phi$ be an automorphism of $G$ leaving both $H$ and $K$ invariant. Give $\mathbb{Z}$ a word metric induced by a generator. Let $L=H \rtimes_{\left.\phi\right|_{H}} \mathbb{Z}$ and $M=G \rtimes_{\phi} \mathbb{Z}$. Then the product metric on $M$ restricted to $H$ is equal to the product metric on $L$ restricted to $H$.

Proof. Let \|\|$_{L}$ and \|\|$_{M}$ the respective norms. Clearly \|\|$_{M} \leq\|\|_{L}$ from the definitions. Let $u \in H$ Suppose $u$ can be written as $u=t^{n_{1}} c_{1} t^{n_{2}} c_{2} \cdots t^{n_{j}} c_{j}$ where $c_{p} \in G$ and $t$ a generator for $\mathbb{Z}$. Let $c_{p}=a_{p}+b_{p}$ where $a_{p} \in H, b_{p} \in K$. By Lemma A.5, $u=v+s$ where $v=t^{n_{1}} a_{1} t^{n_{2}} a_{2} \cdots t^{n_{j}} a_{j}$ and $s=t^{n_{1}} b_{1} t^{n_{2}} b_{2} \cdots t^{n_{j}} b_{j}$. Since $\phi$ fixes both $H$ and $K, v$ lies in $H$ and $s$ lies in $K$. Thus $s=1_{G}$, and so we may 
assume that $\left(b_{p}\right)=\left(1_{G}\right)$. Applying an infimum argument, as in the last result, we are done.

\section{REFERENCES}

[BGS] W. Ballman, M. Gromov and V. Schroeder Manifolds of Nonpositive Curvature, Progress in Mathematics Vol. 61, Birkhäuser (1985).

[B] M. Bestvina, Artin Groups, in preparation.

[Bu] H. Busemann Spaces with Non-positive Curvature, Acta Math 80, 259-310 (1960).

[CS] Chris Croke and Victor Schroeder The fundamental group of compact manifolds without conjugate points, Comm. Math. Helv., 61 (1986) 161-175.

[C1] G. Conner Translation Numbers of Groups acting on Convex Spaces, submitted.

[C2] G. Conner Properties of Translation Numbers in Nilpotent Groups, Comm. Alg., 26(4), 1069-1080 (1998).

[C3] G. Conner A class of finitely generated groups with irrational translation numbers, Arch. Math. 69 (1997), 265-274.

[Gr] M. Gromov Hyperbolic Manifolds, Groups and Actions, Riemann Surfaces and Related Topics, Proceedings of the 1978 Stony Brook Conference, Princeton University Press (1980).

[GW] Detlef Gromoll and Joseph A. Wolf Some Relations Between the Metric Structure and the Algebraic Structure of the Fundamental Group in Manifolds of Nonpositive Curvature, Bul. Amer. Math. Soc. 4 (1971), 545-552.

[GS] S. M. Gersten and H. B. Short Rational Subgroups of Biautomatic Groups, Annals of Math. 134 (1991), 125-158.

[K] Bruce Kleiner, private communication.

[S] Daniel Segal Polycyclic Groups, Cambridge tracts in Mathematics, Cambridge Univ. Press (1983).

Math Department, Brigham Young University, Provo, UT. 84602, USA

E-mail address: conner@math.byu.edu 